\title{
Nonsurgical Therapeutic Options in Portal Vein Thrombosis
}

\author{
Michael Schultheiß Dominik Bettinger Robert Thimme \\ Department of Medicine II, University Hospital Freiburg, Freiburg i.Br., Germany
}

\section{Keywords}

Acute portal vein thrombosis .

Chronic portal vein thrombosis . Portal hypertension . Anticoagulation

\section{Summary}

Background: Portal vein thrombosis (PVT) is a rare but severe vascular disorder with an acute and a chronic course. Most patients have underlying liver cirrhosis; furthermore, thrombophilia is an important risk factor. However, idiopathic forms are also known. Methods: This review discusses nonsurgical treatment options in PVT. Results and Conclusion: Therapy of acute PVT is based on anticoagulation with heparin that is switched to oral anticoagulants, if applicable. Catheter-guided invasive therapy should be considered; however, patients with liver cirrhosis should be screened for portal hypertension before anticoagulation is mandatory. Therapy of chronic PVT is discussed controversially; therefore, a strict patient selection and an individual therapeutic decision are warranted depending on the etiology of PVT. Special forms of PVT including septic and malignant thrombosis as well as PVT in patients waiting for liver transplantation require particular therapy algorithms.

\author{
Schlüsselwörter \\ Akute Pfortaderthrombose . \\ Chronische Pfortaderthrombose - Portale Hypertension . \\ Antikoagulation
}

\section{Zusammenfassung}

Hintergrund: Die Pfortaderthrombose (PAT) ist eine seltene, aber gravierende Gefäßpathologie, die in eine akute und eine chronische Form unterschieden wird. Häufig tritt die PAT bei Patienten mit einer Leberzirrhose auf. Neben idiopathischen Formen sind insbesondere Patienten mit Gerinnungsstörungen betroffen. Methoden: Diese Übersicht beschreibt nichtchirurgische therapeutische Optionen der PAT. Ergebnisse und Schlussfolgerung: Die Behandlung der akuten PAT basiert auf einer Antikoagulation mit Heparin, später gegebenenfalls auch mit oralen Antikoagulanzien. Kathetergeführte invasive Verfahren können zusätzlich erwogen werden. Insbesondere bei Leberzirrhose ist jedoch eine Vordiagnostik bezüglich einer portalen Hypertension unerlässlich. Die Behandlung der chronischen PAT mit Heparin ist umstritten, sodass hier eine strikte Patientenselektion und eine individuelle Therapieentscheidung notwendig sind. Im Rahmen des ätiologischen Kontexts sollten septische und maligne PATs sowie Patienten vor einer Lebertransplantation gesondert betrachtet werden.

\section{Introduction}

Portal vein thrombosis (PVT) is a rare hepatic vascular disorder. It usually occurs in patients with advanced chronic hepatic insufficiency such as decompensated liver cirrhosis; however, it has also been reported in healthy individuals. Risk factors include prothrombotic conditions as well as abdominal inflammation like acute pancreatitis or inflammatory bowel disease. However, idiopathic PVT may also occur. Clinical presentation is very heterogeneous and is linked to the rapidity of thrombus development. Acute PVT can be associated with abdominal pain, dyspepsia, or fever. In contrast, chronic

\section{KARGER \\ Fax +497614520714

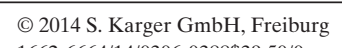

Dr. Michael Schultheiß

Department of Medicine II

University Hospital Freiburg

Hugstetter Straße 55, 79106 Freiburg, Germany

michael.schultheiss@uniklinik-freiburg.de 
Fig. 1. Settings in which PVT occurs and therapeutic recommendations.

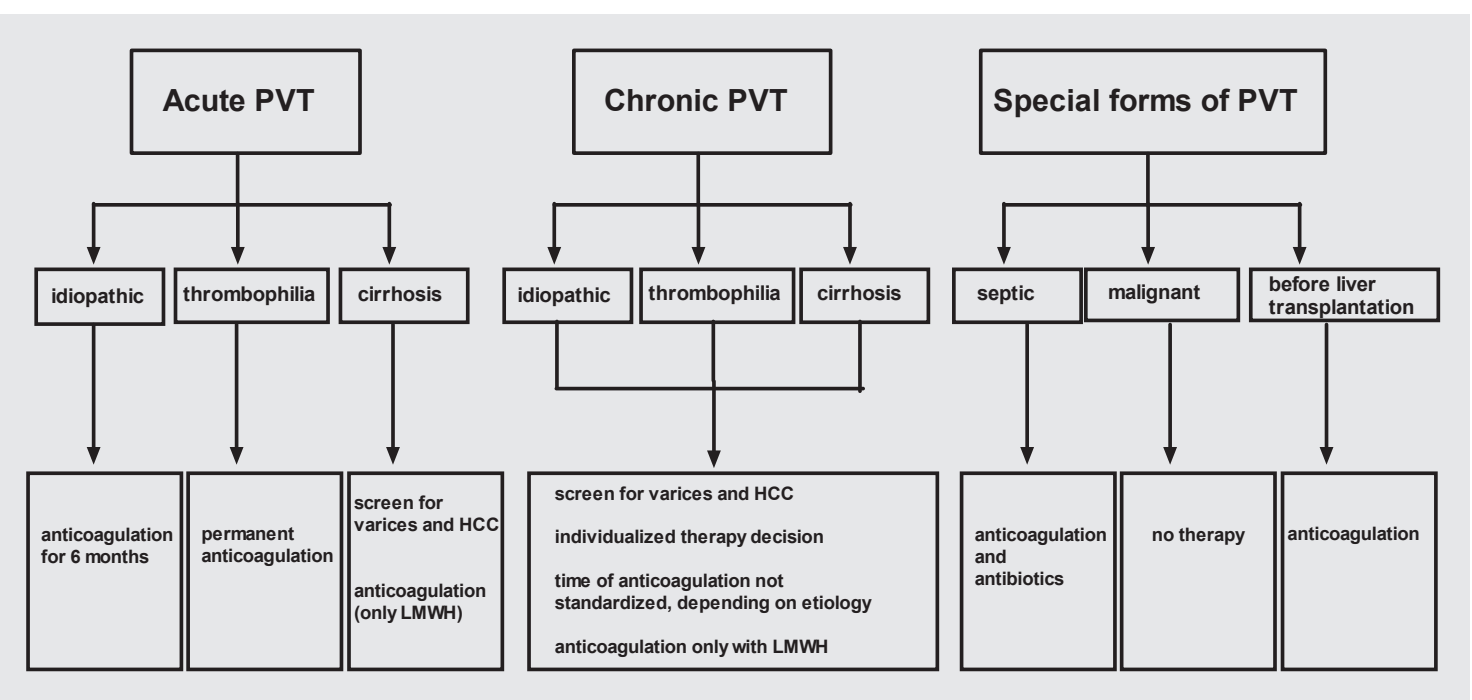

PVT may rather manifest with signs of portal hypertension such as variceal bleeding and/or ascites. Involvement of the superior mesenteric vein (SMV) may lead to an acute abdomen that is caused by mesenterial infarction. It is important to note, though, that acute and more typically chronic PVT may also remain clinically silent.

Diagnosis is primarily based on imaging, i.e., an abdominal ultrasound with Doppler imaging is the first procedure to diagnose PVT. Furthermore, it is based on the detection of a hyperechoic thrombus as well as a limited or absent portal blood flow. Reduced blood flow may indicate portal hypertension. Other possible features include splenomegaly or ascites. Abdominal computed tomography (CT) scan or alternatively magnetic resonance imaging (MRI) should subsequently be performed to determine the complete dimension of the thrombosis and to clarify the etiology (e.g. inflammatory causes like pancreatitis).

The primary nonsurgical therapeutic approach in PVT is in analogy to other vein thromboses - anticoagulation therapy. The special forms of PVT and the patient's condition require a thorough evaluation of the duration and selection of the anticoagulation medication. Systemic or local thrombolytic therapies are possible second-line treatment options even if they are not based on current guidelines.

\section{Settings of Portal Vein Thrombosis}

The choice of optimal therapeutic intervention is based on the diagnosis of the underlying cause of PVT. First, it has to be distinguished between acute and chronic PVT. Second, the etiology has to be determined, and idiopathic PVT, PVT with thrombotic risk factors (state of hypercoagulation), and PVT in liver cirrhosis have to be considered. Special therapeutic issues have to be addressed regarding patients with malignant and septic PVT as well for patients scheduled for liver transplantation (fig. 1).

\section{Acute Portal Vein Thrombosis}

Acute PVT may lead to portal hypertension and, if the SMV is affected, also to intestinal infarction. Spontaneous recanalization is rare, although it has been described in 2 patients with transient inflammatory etiologies of PVT $[1,2]$. Therefore, a sufficient therapy in the form of anticoagulation is recommended. Guidelines from the American Association for the Study of Liver Diseases (AASLD) [3] recommend an initial therapy with low-molecular-weight heparin (LMWH) which is subsequently switched to an oral anticoagulant such as warfarin with a sighted international normalized ratio (INR) of 2-3. Currently, no data is available about novel oral anticoagulant therapy (thrombin inhibitors or direct factor $\mathrm{X}$ inhibitors). Treatment is recommended for at least 3-6 months. An extension of therapy $>6$ months may be considered in cases of severe thrombosis up to the mesenteric vein or if no response to therapy is observed within 6 months. After immediate initiation of therapy higher recanalization rates have been reported with a complete thrombolysis in up to $50 \%$ of the cases [4].

In patients where screening for thrombophilia revealed a permanent thrombotic risk factor, lifelong anticoagulation is recommended.

Treatment of acute PVT in patients with liver cirrhosis has not been defined in guidelines and is discussed controversially. This is surprising considering a PVT prevalence of 10$25 \%$ in patients with liver cirrhosis even in the absence of hepatocellular carcinoma (HCC) [5]. Some studies have reported better recanalization rates in these patients after anticoagulation [6, 7]. In the 9th American College of Chest Physicians (ACCP) Antithrombotic Guidelines, treatment is recommended in symptomatic but not in asymptomatic PVT patients [8]. Prior to initiation of anticoagulation therapy, these patients have to be screened for existing portal hypertension, e.g. esophageal varices or thrombopenia due to hypersplenism, in order to judge the risk of bleeding complications. Ma- 


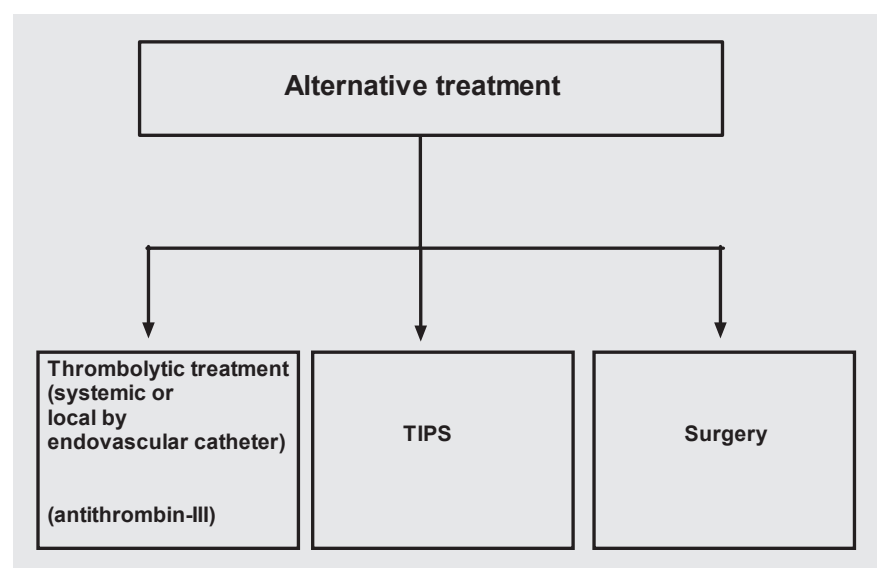

Fig. 2. Alternative therapeutic options in PVT.

lignant thrombosis in HCC is ruled out by using initial diagnostic imaging. Generally, in patients with PVT and cirrhosis, vitamin $\mathrm{K}$ antagonists (VKA) are not used due to prolonged effects and bleeding complications. In addition, the monitoring of VKA therapy by means of INR in cirrhotic patients is called 'unreliable' [9]. In these patients, anticoagulation should be continued with LMWH. Studies showed that LMWH is a safe and effective treatment, with a recanalization rate of up to $80 \%$ and with few episodes of nonsevere variceal bleeding $[7,10,11]$.

In patients not responding to anticoagulation therapy or when the SMV is affected, alternative therapies, such as systemic treatment with streptokinase or tissue plasminogen activators or a catheter-guided local thrombolysis with thrombolytic treatment, may be considered [12] (figs. 2-4). Unfortunately, only very little data has been published about these approaches. However, a high procedure-related complication rate is suspected $[13,14]$. The possible role of placement of the transjugular intrahepatic portosystemic shunt (TIPS) is shown elsewhere in this issue. Other possible future medical approaches include the administration of antithrombin III (AT-III). Indeed, treatment with AT-III showed a preventive effect in cirrhotic patients with PVT after splenectomy [15]. Finally, a surgical approach needs to be considered in the treatment of PVT in patients with intestinal infarction.

\section{Chronic Portal Vein Thrombosis}

Acute PVT may progress to chronic PVT if continuous clotting of the portal (and mesenteric) vein occurs. These patients may develop collateral blood vessels, called cavernous transformation of the portal vein, and frequently suffer from gastrointestinal bleeding caused by esophageal or gastric varices due to portal hypertension.

In contrast to acute PVT, treatment recommendations for chronic PVT are not well established and controversially discussed. In addition, treatment guidelines have not been estab-
Fig. 3. 18-year-old patient with acute PVT in the setting of Crohn's disease. Catheter-guided local lysis with urokinase in the splanchnic vein and superior mesenteric vein.

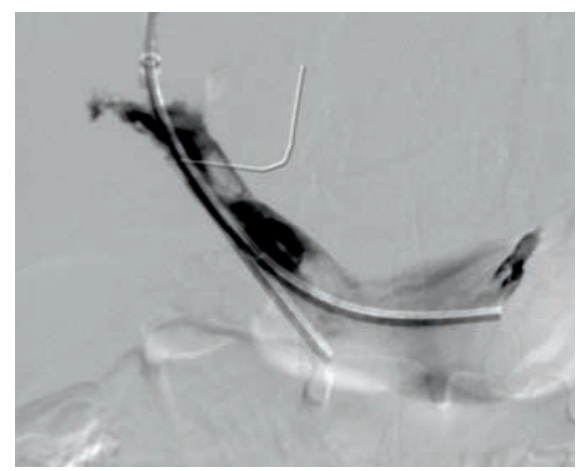

Fig. 4. Recanalization of PVT after 6 days of local lysis.

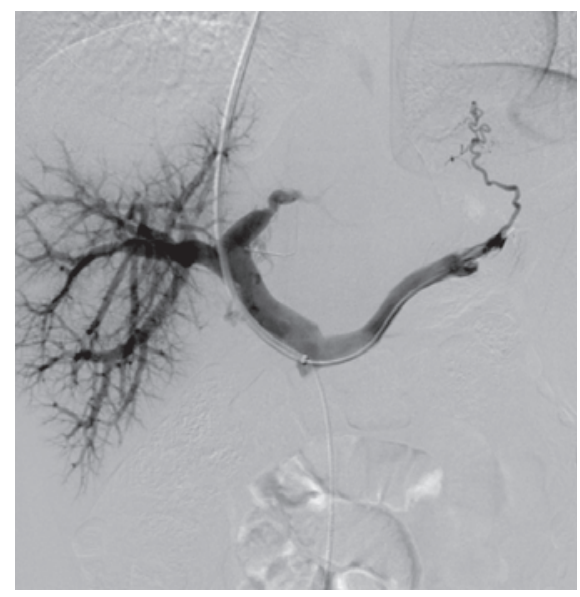

lished for chronic PVT. However, most experts recommend a strict patient selection and individual treatment decision that is based on the etiology of PVT. Importantly, all patients need to be screened (and treated) via endoscopy for esophageal and gastric varices to reduce the risk of intestinal bleeding after the introduction of anticoagulation therapy. Nonselective beta blockers can be useful as a noninvasive prophylaxis in this situation.

Principally, all patients should be considered for anticoagulation therapy since thrombus extension or recurrence may aggravate the status of portal hypertension or lead to intestinal infarction. In addition, (undetected) permanent thrombotic disorders may be the underlying etiology of PVT. As discussed above, in patients with liver cirrhosis and acute PVT, treatment should primarily be based on LMWH since oral VKAs can lead to prolonged dysfunction of anticoagulation in the case of bleeding. Small sample-sized prospective studies as well as a retrospective study showed that anticoagulation in these patients was effective as evidenced by higher recanalization rates and good safety data concerning bleeding complications $[7,10,16]$. Long-term anticoagulation may be considered an option for patients with chronic PVT without cirrhosis or in patients with permanent risk factors for venous thrombosis [3].

Prophylactic anticoagulation to prevent thrombus extension might be reasonable in patients with thrombotic risk factors but high risk of gastrointestinal bleeding. 
Chronic PVT may also lead to portal cholangiopathy. This is caused by venous collateral blood vessels that compress the large bile ducts. Patients with signs of obstructive cholestasis (jaundice, cholangitis) should be treated with endoscopic therapy, such as extraction of bile duct stones and insertion of biliary stents.

\section{Special Forms of Portal Vein Thrombosis}

PVT in the presence of infection may lead to a so-called 'portal pylephlebitis' or septic thrombophlebitis of the portal vein. It is usually caused by abdominal infections drained via the portal vein, such as diverticulitis, appendicitis, or intestinal bowel disease (IBD), and can lead to hepatic abscesses [17]. Therapy includes a broad-spectrum and long-term ( $>6$ weeks) antibiotic coverage $[18,19]$ and in some cases drainage. As recommended by the above-mentioned AASLD guidelines, patients with acute PVT and any signs of infection should be treated with antibiotics [3]. It is of note that cases of recanalization have been described after sole antibiotic therapy $[18,20]$. Still, as described above, systemic anticoagulation for 6 months is recommended.

The incidence of PVT in patients with liver cirrhosis and HCC, therefore called malignant or neoplastic PVT, is high and reaches up to $33 \%$ [5]. The nomenclature 'thrombosis' is not correct in this setting since plugging of the portal vein is primarily caused by an occlusion due to tumor invasion or obstruction. The diagnosis is based on contrast-enhanced imaging, showing contrast injection in the supposed thrombus, or via Doppler ultrasound, indicating a pulsatile flow [21, 22]. Due to the etiology of the thrombus, anticoagulation is not recommended in this setting since tumor tissue cannot be dissolved by anticoagulation, and an elevated bleeding risk has to be considered.

A future therapeutic option in these patients with liver cirrhosis and HCC and therefore with a poor prognosis may be the insertion of a metallic stent to avoid malignant progression of the thrombus from a portal branch to the main portal trunk [23].

Intestinal vein thrombosis has been shown to be a negative prognostic predictor in patients with liver cirrhosis who are on the waiting list for liver transplantation. Especially, the extension of the thrombus to the SMV as well as a complete PVT has been shown to be associated with an inferior outcome in short-term observations after transplantation [24, 25]. In one study, patients with splanchnic vein thrombosis treated with anticoagulation showed significantly higher recanalization rates [25]. Therefore, anticoagulation treatment in patients with liver cirrhosis on the waiting list for liver transplantation and in those with PVT is recommended [26-28].

\section{Conclusion}

In acute PVT, immediate anticoagulation treatment is the first and main therapy component. Special attention has to be given to patients with liver cirrhosis and bleeding complications. In advanced thrombosis up to the mesenteric vein, aggressive conservative treatment with thrombolysis, AT-III, or invasive therapies with local catheter-guided lysis or TIPS might be needed. The duration of anticoagulation treatment has to be determined by the underlying coagulation disorder.

Treatment recommendations for chronic PVT have not been clearly established due to the 'chronic' occurrence of clotting and the cavernous transformation of the portal vein, while the success of anticoagulation is still discussed controversially. Furthermore, the underlying portal hypertension with varices and thrombopenia constitutes a risk factor for anticoagulation in these patients. We believe that treatment should be started after an initial diagnostic procedure consisting of gastroscopy and endoscopic/medical treatment of varices as well as a strict patient selection.

Next to anticoagulation therapy, antibiotic treatment is the state of the art in septic PVT or PVT with any suspicion of infection. Malignant PVT should not be treated by anticoagulation since no recanalization can be expected due to the etiology and bleeding complications are frequent. The indication for anticoagulation in patients waiting for liver transplantation is strong since thrombotic events are a major risk factor for peri- and postoperative complications in liver transplantation.

\section{Disclosure Statement}

The authors do not have any conflicts of interest regarding this article.

\section{References}

1 Plessier A, Darwish-Murad S, Hernandez-Guerra M, Consigny Y, Fabris F, Trebicka J, Heller J, Morard I, Lasser L, Langlet P, Denninger MH, Vidaud D, Condat B, Hadengue A, Primignani M, Garcia-Pagan JC, Janssen HL, Valla D: Acute portal vein thrombosis unrelated to cirrhosis: a prospective multicenter follow-up study. Hepatology 2010;51:210-218.

\footnotetext{
2 Davies M, Satyadas T, Akle CA: Spontaneous resolution of a superior mesenteric vein thrombosis after laparoscopic Nissen fundoplication. Ann R Coll Surg Engl 2002;84:177-180.

3 DeLeve LD, Valla DC, Garcia-Tsao G: Vascular
disorders of the liver. Hepatology 2009;49:17291764.
}

4 Condat B, Pessione F, Helene Denninger M, Hillaire S, Valla D: Recent portal or mesenteric venous thrombosis: increased recognition and frequent recanalization on anticoagulant therapy. Hepatology 2000;32:466-470.

5 Nonami T, Yokoyama I, Iwatsuki S, Starzl TE: The incidence of portal vein thrombosis at liver transplantation. Hepatology 1992;16:1195-1198. 
6 Delgado MG, Seijo S, Yepes I, Achecar L, Catalina MV, Garcia-Criado A, Abraldes JG, de la Pena J, Banares R, Albillos A, Bosch J, Garcia-Pagan JC: Efficacy and safety of anticoagulation on patients with cirrhosis and portal vein thrombosis. Clin Gastroenterol Hepatol 2012;10:776-783.

7 Senzolo M, Sartori MT, Rossetto V, Burra P, Cillo U, Boccagni P, Gasparini D, Miotto D, Simioni P, Tsochatzis E, Burroughs AK: Prospective evaluation of anticoagulation and transjugular intrahepatic portosystemic shunt for the management of portal vein thrombosis in cirrhosis. Liver Int 2012; 32:919-927.

8 Kearon C, Akl EA, Comerota AJ, Prandoni P, Bounameaux H, Goldhaber SZ, Nelson ME, Wells PS, Gould MK, Dentali F, Crowther M, Kahn SR; American College of Chest Physicians: Antithrombotic therapy for VTE disease: Antithrombotic Therapy and Prevention of Thrombosis, 9th ed: American College of Chest Physicians EvidenceBased Clinical Practice Guidelines. Chest 2012; 141:e419S-494S

-9 Arjal R, Trotter JF: International normalized ratio of prothrombin time in the model for end-stage liver disease score: an unreliable measure. Clin Liver Dis 2009;13:67-71.

10 Amitrano L, Guardascione MA, Menchise A, Martino R, Scaglione M, Giovine S, Romano L, Balzano A: Safety and efficacy of anticoagulation therapy with low molecular weight heparin for portal vein thrombosis in patients with liver cirrhosis. $\mathbf{J}$ Clin Gastroenterol 2010;44:448-451.

-11 Senzolo M, Ferronato C, Burra P, Sartori MT: Anticoagulation for portal vein thrombosis in cirrhotic patients should be always considered. Intern Emerg Med 2009;4:161-162; author reply 163-164.

12 Malkowski P, Pawlak J, Michalowicz B, Szczerban J, Wroblewski T, Leowska E, Krawczyk M: Thrombolytic treatment of portal thrombosis. Hepatogastroenterology 2003;50:2098-2100.
13 Hollingshead M, Burke CT, Mauro MA, Weeks SM, Dixon RG, Jaques PF: Transcatheter thrombolytic therapy for acute mesenteric and portal vein thrombosis. J Vasc Interv Radiol 2005;16: 651-661.

14 Grisham A, Lohr J, Guenther JM, Engel AM: Deciphering mesenteric venous thrombosis: imaging and treatment. Vasc Endovascular Surg 2005;39: 473-479.

15 Kawanaka H, Akahoshi T, Kinjo N, Konishi K, Yoshida D, Anegawa G, Yamaguchi S, Uehara H, Hashimoto N, Tsutsumi N, Tomikawa M, Maehara Y: Impact of antithrombin III concentrates on portal vein thrombosis after splenectomy in patients with liver cirrhosis and hypersplenism. Ann Surg 2010;251:76-83.

16 Condat B, Pessione F, Hillaire S, Denninger MH, Guillin MC, Poliquin M, Hadengue A, Erlinger S, Valla D: Current outcome of portal vein thrombosis in adults: risk and benefit of anticoagulant therapy. Gastroenterology 2001;120:490-497.

17 Aguas M, Bastida G, Nos P, Beltran B, Grueso JL, Grueso J: Septic thrombophlebitis of the superior mesenteric vein and multiple liver abscesses in a patient with Crohn's disease at onset. BMC Gastroenterol 2007;7:22.

18 Plemmons RM, Dooley DP, Longfield RN: Septic thrombophlebitis of the portal vein (pylephlebitis): diagnosis and management in the modern era. Clin Infect Dis 1995;21:1114-1120.

19 Singh P, Yadav N, Visvalingam V, Indaram A, Bank S: Pylephlebitis - diagnosis and management. Am J Gastroenterol 2001;96:1312-1313.

20 Baril N, Wren S, Radin R, Ralls P, Stain S: The role of anticoagulation in pylephlebitis. Am J Surg 1996;172:449-452; discussion 452-443.

21 Tublin ME, Dodd GD 3rd, Baron RL: Benign and malignant portal vein thrombosis: differentiation by CT characteristics. AJR Am J Roentgenol 1997; 168:719-723.
2 Rossi S, Rosa L, Ravetta V, Cascina A, Quaretti P, Azzaretti A, Scagnelli P, Tinelli C, Dionigi P, Calliada F: Contrast-enhanced versus conventional and color Doppler sonography for the detection of thrombosis of the portal and hepatic venous systems. AJR Am J Roentgenol 2006;186:763-773.

23 Vibert E, Azoulay D, Cunha AS, Adam R, Samuel D, Castaing D: Portal stenting for hepatocellular carcinoma extending into the portal vein in cirrhotic patients. J Surg Oncol 2013;107:696-701.

24 Rodriguez-Castro KI, Porte RJ, Nadal E, Germani G, Burra P, Senzolo M: Management of nonneoplastic portal vein thrombosis in the setting of liver transplantation: a systematic review. Transplantation 2012;94:1145-1153.

25 Francoz C, Belghiti J, Vilgrain V, Sommacale D, Paradis V, Condat B, Denninger MH, Sauvanet A Valla D, Durand F: Splanchnic vein thrombosis in candidates for liver transplantation: usefulness of screening and anticoagulation. Gut 2005;54:691-697.

26 Lendoire J, Raffin G, Cejas N, Duek F, Barros Schelotto P, Trigo P, Quarin C, Garay V, Imventarza O: Liver transplantation in adult patients with portal vein thrombosis: risk factors, management and outcome. HPB (Oxford) 2007;9:352-356.

27 Selvaggi G, Weppler D, Nishida S, Moon J, Levi D, Kato T, Tzakis AG: Ten-year experience in portocaval hemitransposition for liver transplantation in the presence of portal vein thrombosis. Am J Transplant 2007;7:454-460.

28 Werner KT, Sando S, Carey EJ, Vargas HE, Byrne TJ, Douglas DD, Harrison ME, Rakela J, Aqel BA: Portal vein thrombosis in patients with end stage liver disease awaiting liver transplantation: outcome of anticoagulation. Dig Dis Sci 2013;58 1776-1780 